\title{
ON ALMOST INCREASING SEQUENCES AND ITS APPLICATIONS
}

\section{HIKMET S. ÖZARSLAN}

(Received 7 July 1999 and in revised form 18 April 2000)

ABSTRACT. We prove a general theorem on $\left|\bar{N}, p_{n} ; \delta\right|_{k}$ summability factors, which generalizes a theorem of Bor (1994) on $\left|\bar{N}, p_{n}\right|_{k}$ summability factors, under weaker conditions by using an almost increasing sequence.

2000 Mathematics Subject Classification. Primary 40D15, 40F05, 40 G99.

1. Introduction. Let $\sum a_{n}$ be a given infinite series with partial sums $\left(s_{n}\right)$. Let $\left(p_{n}\right)$ be a sequence of positive numbers such that

$$
P_{n}=\sum_{v=0}^{n} p_{v} \longrightarrow \infty \quad \text { as } n \longrightarrow \infty, \quad\left(P_{-i}=p_{-i}=0, i \geq 1\right)
$$

The sequence-to-sequence transformation

$$
T_{n}=\frac{1}{P_{n}} \sum_{v=0}^{n} p_{v} s_{v}
$$

defines the sequence $\left(T_{n}\right)$ of the $\left(\bar{N}, p_{n}\right)$ mean of the sequence $\left(s_{n}\right)$, generated by the sequence of coefficients ( $\left.p_{n}\right)$ (see [4]). The series $\sum a_{n}$ is said to be summable $\left|\bar{N}, p_{n}\right|_{k}$, $k \geq 1$, if (see [1])

$$
\sum_{n=1}^{\infty}\left(\frac{P_{n}}{p_{n}}\right)^{k-1}\left|\Delta T_{n-1}\right|^{k}<\infty
$$

and it is said to be summable $\left|\bar{N}, p_{n} ; \delta\right|_{k}, k \geq 1$ and $\delta \geq 0$, if (see [2])

$$
\sum_{n=1}^{\infty}\left(\frac{P_{n}}{p_{n}}\right)^{\delta k+k-1}\left|\Delta T_{n-1}\right|^{k}<\infty
$$

where

$$
\Delta T_{n-1}=-\frac{p_{n}}{P_{n} P_{n-1}} \sum_{v=1}^{n} P_{v-1} a_{v}, \quad n \geq 1
$$

In the special case when $\delta=0$, (respectively, $p_{n}=1$ for all values of $n$ ) $\left|\bar{N}, p_{n} ; \delta\right|_{k}$ summability is the same as $\left|\bar{N}, p_{n}\right|_{k}$ (respectively, $|C, 1 ; \delta|_{k}$ ) summability. 
Mishra and Srivastava [6] proved the following theorem for $|C, 1|_{k}$ summability.

THEOREM 1.1. Let $\left(X_{n}\right)$ be a positive nondecreasing sequence and let there be sequences $\left(\beta_{n}\right)$ and $\left(\lambda_{n}\right)$ such that

$$
\begin{gathered}
\left|\Delta \lambda_{n}\right| \leq \beta_{n}, \\
\beta_{n} \longrightarrow 0 \quad \text { as } n \longrightarrow \infty, \\
\left|\lambda_{n}\right| X_{n}=O(1) \quad \text { as } n \rightarrow \infty \\
\sum_{n=1}^{\infty} n\left|\Delta \beta_{n}\right| X_{n}<\infty .
\end{gathered}
$$

If

$$
\sum_{n=1}^{m} \frac{1}{n}\left|s_{n}\right|^{k}=O\left(X_{m}\right) \quad \text { as } m \longrightarrow \infty,
$$

then the series $\sum a_{n} \lambda_{n}$ is summable $|C, 1|_{k}, k \geq 1$.

Bor [3] has generalized Theorem 1.1 for $\left|\bar{N}, p_{n}\right|_{k}$ summability in the form of the following theorem.

THEOREM 1.2. Let $\left(X_{n}\right)$ be a positive nondecreasing sequence and the sequences $\left(\beta_{n}\right)$ and $\left(\lambda_{n}\right)$ such that conditions (1.6), (1.7), (1.8), and (1.9) of Theorem 1.1 are satisfied. Furthermore, if $\left(p_{n}\right)$ is a sequence of positive numbers such that

$$
\begin{gathered}
P_{n}=O\left(n p_{n}\right) \quad \text { as } n \rightarrow \infty, \\
\sum_{n=1}^{m} \frac{p_{n}}{P_{n}}\left|s_{n}\right|^{k}=O\left(X_{m}\right) \quad \text { as } m \longrightarrow \infty,
\end{gathered}
$$

then the series $\sum a_{n} \lambda_{n}$ is summable $\left|\bar{N}, p_{n}\right|_{k}, k \geq 1$.

It should be noted that, if we take $p_{n}=1$ for all values of $n$, then condition (1.12) will be reduced to condition (1.10). Also, it can be noticed that in this case condition (1.11) is obvious.

2. The main result. The aim of this paper is to generalize Theorem 1.2 for $\left|\bar{N}, p_{n} ; \delta\right|_{k}$ summability under weaker conditions. For this we need the concept of almost increasing sequence. A positive sequence $\left(b_{n}\right)$ is said to be almost increasing if there exists a positive increasing sequence $\left(c_{n}\right)$ and two positive constants $A$ and $B$ such that $A c_{n} \leq b_{n} \leq B c_{n}$. Obviously every increasing sequence is almost increasing sequence but the converse need not be true as can be seen from the example $b_{n}=n e^{(-1)^{n}}$. So we are weakening the hypotheses of Theorem 1.2 replacing the increasing sequence by an almost increasing sequence.

Now, we will prove the following theorem.

THEOREM 2.1. Let $\left(X_{n}\right)$ be an almost increasing sequence and the sequences $\left(\beta_{n}\right)$ and $\left(\lambda_{n}\right)$ such that conditions (1.6), (1.7), (1.8), and (1.9) of Theorem 1.1 are satisfied. 
If $\left(p_{n}\right)$ is a sequence such that condition (1.11) of Theorem 1.2 is satisfied and

$$
\begin{aligned}
\sum_{n=1}^{m}\left(\frac{P_{n}}{p_{n}}\right)^{\delta k-1}\left|s_{n}\right|^{k} & =O\left(X_{m}\right) \quad \text { as } m \rightarrow \infty, \\
\sum_{n=v+1}^{\infty}\left(\frac{P_{n}}{p_{n}}\right)^{\delta k-1} \frac{1}{P_{n-1}} & =O\left(\left(\frac{P_{v}}{p_{v}}\right)^{\delta k} \frac{1}{P_{v}}\right),
\end{aligned}
$$

then the series $\sum a_{n} \lambda_{n}$ is summable $\left|\bar{N}, p_{n} ; \delta\right|_{k}$ for $k \geq 1$ and $0 \leq \delta<1 / k$.

REMARK 2.2. It may be noted that if we take $\left(X_{n}\right)$ as a positive nondecreasing sequence and $\delta=0$ in this theorem, then we get Theorem 1.2. In this case, condition (2.1) reduces to condition (1.12) and condition (2.2) reduces to

$$
\sum_{n=v+1}^{\infty} \frac{p_{n}}{P_{n} P_{n-1}}=O\left(\frac{1}{P_{v}}\right),
$$

which always holds.

We need the following lemma for the proof of our theorem.

LEMMA 2.3 (see [5]). Under the conditions on $\left(X_{n}\right),\left(\beta_{n}\right)$, and $\left(\lambda_{n}\right)$ as taken in the statement of the theorem, the following conditions hold, when (1.9) is satisfied:

$$
\begin{gathered}
n \beta_{n} X_{n}=O(1) \quad \text { as } n \longrightarrow \infty, \\
\sum_{n=1}^{\infty} \beta_{n} X_{n}<\infty .
\end{gathered}
$$

Proof of Theorem 2.1. Let $\left(T_{n}\right)$ denotes the $\left(\bar{N}, p_{n}\right)$ mean of the series $\sum a_{n} \lambda_{n}$. Then, by definition and changing the order of summation, we have

$$
T_{n}=\frac{1}{P_{n}} \sum_{v=0}^{n} p_{v} \sum_{i=0}^{v} a_{i} \lambda_{i}=\frac{1}{P_{n}} \sum_{v=0}^{n}\left(P_{n}-P_{v-1}\right) a_{v} \lambda_{v} .
$$

Then, for $n \geq 1$, we have

$$
T_{n}-T_{n-1}=\frac{p_{n}}{P_{n} P_{n-1}} \sum_{v=1}^{n} P_{v-1} a_{v} \lambda_{v} .
$$

By Abel's transformation, we have

$$
\begin{aligned}
T_{n}-T_{n-1} & =\frac{p_{n}}{P_{n} P_{n-1}} \sum_{v=1}^{n-1} \Delta\left(P_{v-1} \lambda_{v}\right) s_{v}+\frac{p_{n}}{P_{n}} s_{n} \lambda_{n} \\
& =-\frac{p_{n}}{P_{n} P_{n-1}} \sum_{v=1}^{n-1} p_{v} s_{v} \lambda_{v}+\frac{p_{n}}{P_{n} P_{n-1}} \sum_{v=1}^{n-1} P_{v} s_{v} \Delta \lambda_{v}+\frac{p_{n}}{P_{n}} s_{n} \lambda_{n} \\
& =T_{n, 1}+T_{n, 2}+T_{n, 3},
\end{aligned}
$$


where $T_{n, i}, i=1,2,3$, denotes the $i$ th term in the sum. Since

$$
\left|T_{n, 1}+T_{n, 2}+T_{n, 3}\right|^{k} \leq 3^{k}\left(\left|T_{n, 1}\right|^{k}+\left|T_{n, 2}\right|^{k}+\left|T_{n, 3}\right|^{k}\right),
$$

to complete the proof of the theorem, it is enough to show that

$$
\sum_{n=1}^{\infty}\left(\frac{P_{n}}{p_{n}}\right)^{\delta k+k-1}\left|T_{n, r}\right|^{k}<\infty \quad \text { for } r=1,2,3 .
$$

Now, when $k>1$ applying Hölder's inequality with indices $k$ and $k^{\prime}$, where $1 / k+1 / k^{\prime}=$ 1 , we have

$$
\begin{aligned}
& \sum_{n=2}^{m+1}\left(\frac{P_{n}}{p_{n}}\right)^{\delta k+k-1}\left|T_{n, 1}\right|^{k} \leq \sum_{n=2}^{m+1}\left(\frac{P_{n}}{p_{n}}\right)^{\delta k-1}\left(P_{n-1}\right)^{-k}\left(\sum_{v=1}^{n-1} p_{v}\left|s_{v}\right|\left|\lambda_{v}\right|\right)^{k} \\
& \leq \sum_{n=2}^{m+1}\left(\frac{P_{n}}{p_{n}}\right)^{\delta k-1} \frac{1}{P_{n-1}} \sum_{v=1}^{n-1} p_{v}\left|s_{v}\right|^{k}\left|\lambda_{v}\right|^{k}\left(\frac{1}{P_{n-1}} \sum_{v=1}^{n-1} p_{v}\right)^{k-1} \\
& =O(1) \sum_{v=1}^{m} p_{v}\left|s_{v}\right|^{k}\left|\lambda_{v}\right|^{k} \sum_{n=v+1}^{m+1}\left(\frac{P_{n}}{p_{n}}\right)^{\delta k-1} \frac{1}{P_{n-1}} \\
& =O(1) \sum_{v=1}^{m}\left(\frac{P_{v}}{p_{v}}\right)^{\delta k-1}\left|s_{v}\right|^{k}\left|\lambda_{v}\right|\left|\lambda_{v}\right|^{k-1} \\
& =O(1) \sum_{v=1}^{m}\left(\frac{P_{v}}{p_{v}}\right)^{\delta k-1}\left|s_{v}\right|^{k}\left|\lambda_{v}\right| \\
& =O(1) \sum_{v=1}^{m-1} \Delta\left|\lambda_{v}\right| \sum_{r=1}^{v}\left(\frac{P_{r}}{p_{r}}\right)^{\delta k-1}\left|s_{r}\right|^{k} \\
& +O(1)\left|\lambda_{m}\right| \sum_{v=1}^{m}\left(\frac{P_{v}}{p_{v}}\right)^{\delta k-1}\left|s_{v}\right|^{k} \\
& =O(1) \sum_{v=1}^{m-1}\left|\Delta \lambda_{v}\right| X_{v}+O(1)\left|\lambda_{m}\right| X_{m} \\
& =O(1) \sum_{v=1}^{m-1} \beta_{v} X_{v}+O(1)\left|\lambda_{m}\right| X_{m} \\
& =O(1) \text { as } m \longrightarrow \infty \text {, }
\end{aligned}
$$

by virtue of the hypotheses of Theorem 1.2 and Lemma 2.3 .

Since $v \beta_{v}=O\left(1 / X_{v}\right)$ by (2.4), using the fact that $P_{v}=O\left(v p_{v}\right)$ by (1.11), and $\left|\Delta \lambda_{n}\right| \leq \beta_{n}$ by (1.6), and after applying Hölder's inequality again, we have 


$$
\begin{aligned}
& \sum_{n=2}^{m+1}\left(\frac{P_{n}}{p_{n}}\right)^{\delta k+k-1}\left|T_{n, 2}\right|^{k} \leq \sum_{n=2}^{m+1}\left(\frac{P_{n}}{p_{n}}\right)^{\delta k-1}\left(P_{n-1}\right)^{-k}\left(\sum_{v=1}^{n-1} P_{v}\left|\Delta \lambda_{v}\right|\left|s_{v}\right|\right)^{k} \\
& =O(1) \sum_{n=2}^{m+1}\left(\frac{P_{n}}{p_{n}}\right)^{\delta k-1}\left(P_{n-1}\right)^{-k}\left(\sum_{v=1}^{n-1} v p_{v} \beta_{v}\left|s_{v}\right|\right)^{k} \\
& =O(1) \sum_{n=2}^{m+1}\left(\frac{P_{n}}{p_{n}}\right)^{\delta k-1} \frac{1}{P_{n-1}} \sum_{v=1}^{n-1}\left(v \beta_{v}\right)^{k} p_{v}\left|s_{v}\right|^{k}\left(\frac{1}{P_{n-1}} \sum_{v=1}^{n-1} p_{v}\right)^{k-1} \\
& =O(1) \sum_{v=1}^{m}\left(v \beta_{v}\right)^{k} p_{v}\left|s_{v}\right|^{k} \sum_{n=v+1}^{m+1}\left(\frac{P_{n}}{p_{n}}\right)^{\delta k-1} \frac{1}{P_{n-1}} \\
& =O(1) \sum_{v=1}^{m}\left(v \beta_{v}\right)^{k}\left(\frac{P_{v}}{p_{v}}\right)^{\delta k-1}\left|s_{v}\right|^{k} \\
& =O(1) \sum_{v=1}^{m}\left(v \beta_{v}\right)^{k-1}\left(v \beta_{v}\right)\left(\frac{P_{v}}{p_{v}}\right)^{\delta k-1}\left|s_{v}\right|^{k} \\
& =O(1) \sum_{v=1}^{m}\left(v \beta_{v}\right)\left(\frac{P_{v}}{p_{v}}\right)^{\delta k-1}\left|s_{v}\right|^{k} \\
& =O(1) \sum_{v=1}^{m-1} \Delta\left(v \beta_{v}\right) \sum_{r=1}^{v}\left(\frac{P_{r}}{p_{r}}\right)^{\delta k-1}\left|s_{r}\right|^{k} \\
& +O(1) m \beta_{m} \sum_{v=1}^{m}\left(\frac{P_{v}}{p_{v}}\right)^{\delta k-1}\left|s_{v}\right|^{k} \\
& =O(1) \sum_{v=1}^{m-1}\left|\Delta\left(v \beta_{v}\right)\right| X_{v}+O(1) m \beta_{m} X_{m} \\
& =O(1) \sum_{v=1}^{m-1} v\left|\Delta \beta_{v}\right| X_{v}+O(1) \sum_{v=1}^{m-1} \beta_{v+1} X_{v+1}+O(1) m \beta_{m} X_{m} \\
& =O(1) \text { as } m \longrightarrow \infty \text {, }
\end{aligned}
$$

by virtue of the hypotheses of Theorem 1.2 and Lemma 2.3 .

Finally, using the fact that $P_{v}=O\left(v p_{v}\right)$ by (1.11), as in $T_{n, 1}$, we have

$$
\begin{aligned}
\sum_{n=1}^{m}\left(\frac{P_{n}}{p_{n}}\right)^{\delta k+k-1}\left|T_{n, 3}\right|^{k} & =O(1) \sum_{n=1}^{m}\left(\frac{P_{n}}{p_{n}}\right)^{\delta k-1}\left|s_{n}\right|^{k}\left|\lambda_{n}\right| \\
& =O(1) \quad \text { as } m \longrightarrow \infty .
\end{aligned}
$$

Therefore, we get (2.10) and this completes the proof of the theorem.

If we take $p_{n}=1$ for all values of $n$ in this theorem, then we get a result concerning the $|C, 1 ; \delta|_{k}$ summability factors. 


\section{REFERENCES}

[1] H. Bor, On two summability methods, Math. Proc. Cambridge Philos. Soc. 97 (1985), no. 1, 147-149. MR 86d:40004. Zbl 554.40008.

[2] _ On local property of $\left|\bar{N}, p_{n} ; \delta\right|_{k}$ summability of factored Fourier series, J. Math. Anal. Appl. 179 (1993), no. 2, 646-649. MR 95a:42002. Zbl 797.42005.

[3] _ A note on absolute summability factors, Int. J. Math. Math. Sci. 17 (1994), no. 3, 479-482. MR 95b:40007. Zbl 802.40004.

[4] G. H. Hardy, Divergent Series, Oxford, at the Clarendon Press, 1949. MR 11,25a. Zbl 032.05801.

[5] S. M. Mazhar, A note on absolute summability factors, Bull. Inst. Math. Acad. Sinica 25 (1997), no. 3, 233-242. MR 99a:40005. Zbl 885.40004.

[6] K. N. Mishra and R. S. L. Srivastava, On absolute Cesàro summability factors of infinite series, Portugal. Math. 42 (1983/84), no. 1, 53-61 (1985). MR 87a:40003. Zbl 597.40003.

HiKMET S. ÖZARSLAN: DEPARTMENT OF MATHEMATICS, ERCIYES UNIVERSITY 38039, KAYSERI, TURKEY

E-mail address: seyhan@erciyes.edu.tr 


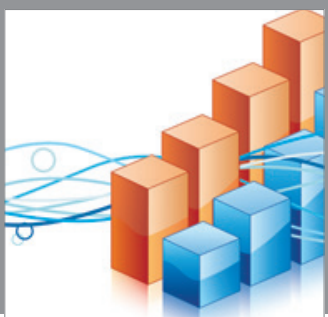

Advances in

Operations Research

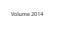

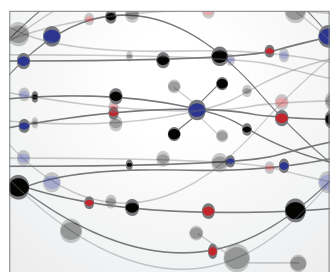

\section{The Scientific} World Journal
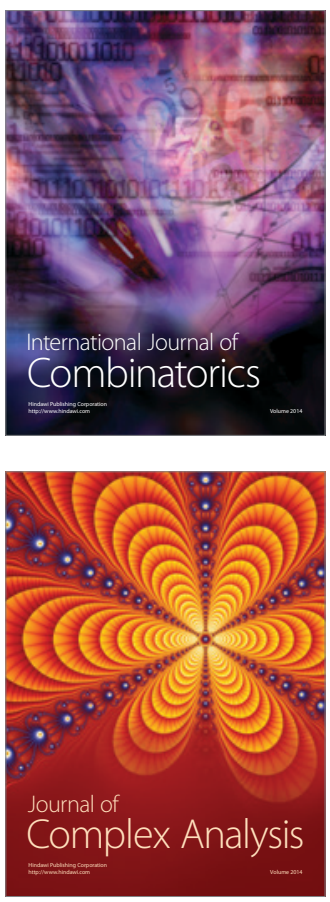

International Journal of

Mathematics and

Mathematical

Sciences
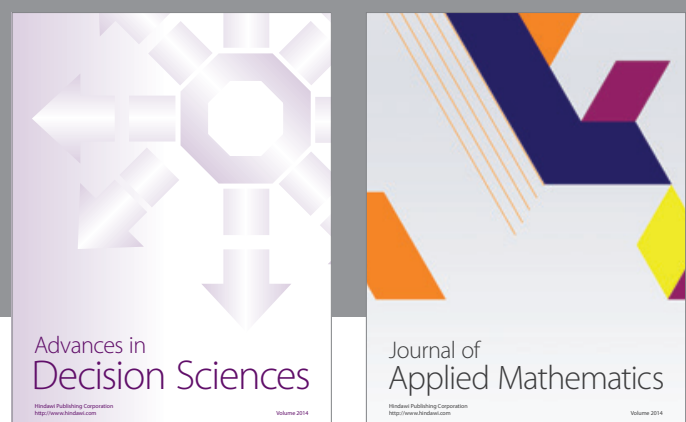

Journal of

Applied Mathematics
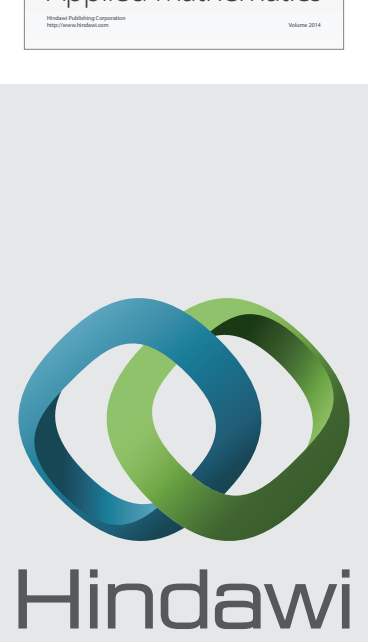

Submit your manuscripts at http://www.hindawi.com
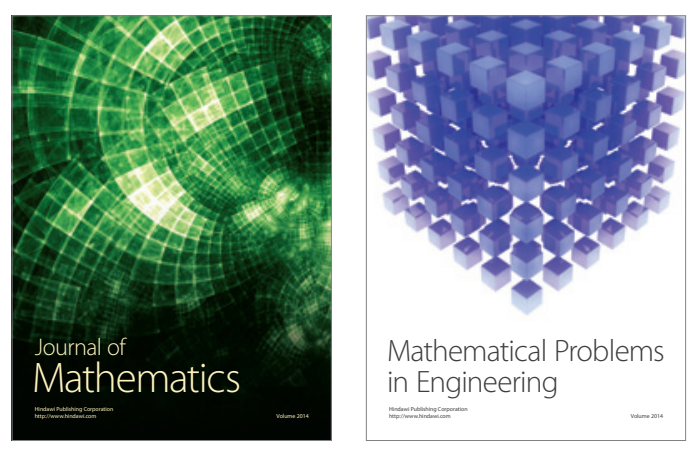

Mathematical Problems in Engineering
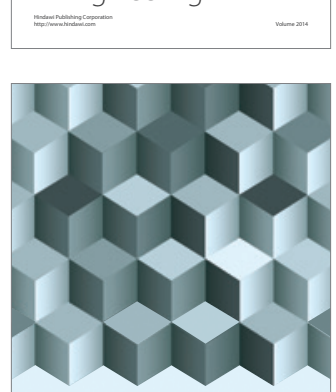

Journal of

Function Spaces
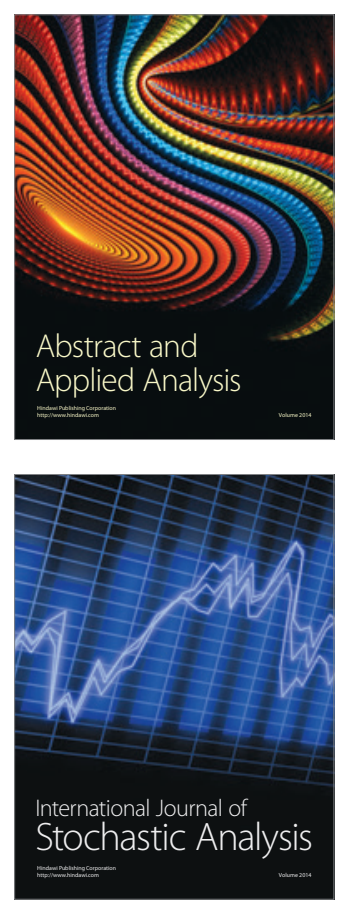

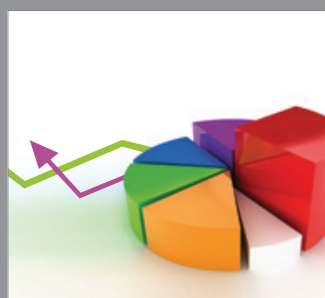

ournal of

Probability and Statistics

Promensencen
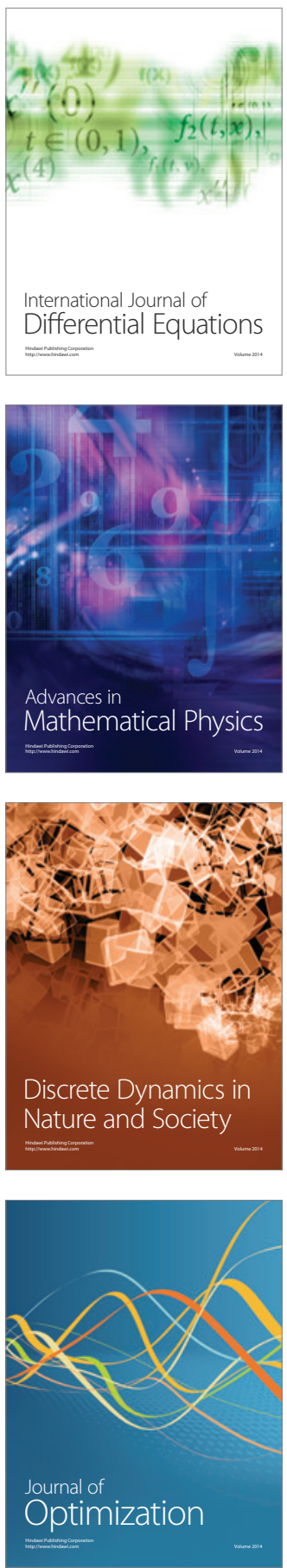